\title{
Phenomenology of the superconducting state of a marginal Fermi liquid with BCS model interaction
}

\author{
M. L. Horbach, ${ }^{*}$ F. L. J. Vos, and W. van Saarloos \\ Institute-Lorentz for Theoretical Physics, University of Leiden, P.O. Box 9506, 2300 RA Leiden, The Netherlands
}

(Received 24 February 1993)

\begin{abstract}
We study the effect of an additional simple BCS model interaction in a system in which the dominant scattering causes marginal-Fermi-liquid (MFL) behavior. It leads to a superconductor that shows a number of unusual features as compared to weak-coupling BCS superconductors. The superconducting transition temperature is lowered due to the MFL pair-breaking effects. If the bosonic mode that causes the MFL behavior, and which presumably is of electronic origin, is largely suppressed below $T_{c}$ due to the gap opening up, $\Delta(0)$ remains practically unaffected by the MFL behavior. As a result, the ratio $2 \Delta(0) / k_{B} T_{c}$ is enhanced, and the gap opens up much more rapidly than in the standard BCS approximation; even a gap discontinuity at $T_{c}$ is possible. Furthermore, we find a suppression of the coherence peak in the nuclear-spin-relaxation rate and a more dramatic decrease below $T_{c}$ of the ultrasonic attenuation rate and the penetration depth.
\end{abstract}

\section{INTRODUCTION}

The unconventional normal-state behavior of the high-temperature superconductors, as compared to normal metals, is well described by the marginal-Fermiliquid (MFL) hypothesis. ${ }^{1}$ Most of the anomalous normal-state properties can, according to this hypothesis, be understood from a single assumption about the polarizability of the (strongly interacting) system, namely,

$$
\operatorname{Im} P(\mathbf{q}, \omega) \propto \tanh (\omega / T) .
$$

It leads for instance to an electronic scattering rate

$$
1 / \tau=(\lambda \pi / 2) \max (T,|\omega|),
$$

where $\lambda=g^{2} N(0)^{2}$, with $g$ being the coupling of the electrons with the unidentified excitations that lead to the unusual polarizability and $N(0)$ the density of states at the Fermi energy.

Also the superconducting state of the hightemperature superconductors shows a number of unusual features in comparison with weak-coupling BCS superconductors. Most prominent are the absence of coherence peaks in the nuclear-spin-relaxation rate $1 / T_{1} T$ (Ref. 2) and in the conductivity $\sigma_{1}(\omega)$ (Ref. 3) (though in the latter quantity peaks arising from lifetime effects or thermal fluctuations can appear), ${ }^{4}$ and an unusually large ratio $2 \Delta(0) / k_{B} T_{c}$, for which values up to 10 have been reported. ${ }^{5}$

The assumption that the dominant scattering occurs by exchange of the excitations that give rise to the unusual polarizability immediately leads to the observation that below $T_{c}$, as the gap opens up, this scattering process will be suppressed considerably (most dramatically if the coupling of the electrons to the polarizability is such that it leads to type-I coherence factors). As a consequence the quasiparticle lifetime will rapidly increase as the temperature is lowered. Measurements of the surface resistance of $\mathrm{YBa}_{2} \mathrm{Cu}_{3} \mathrm{O}_{7}$ (Ref. 6) do indeed appear to give evidence for a suppression of the quasiparticle scattering rate, and hence support the assumption about the dominant role of an electronic scattering process involving a nontrivial renormalization of the polarizability.

It is aesthetically most attractive to assume that the (yet unknown) physics underlying the MFL hypothesis is also the origin of the superconductivity. ${ }^{7}$ Kuroda and Varma $^{8}$ and Littlewood and Varma ${ }^{9}$ have pursued this idea in an Eliashberg strong-coupling approach, in which the MFL polarizability plays the role of the bosonic mode that is responsible for the superconductivity, i.e., where the MFL polarizability replaces the usual electron-phonon coupling $\alpha^{2} F(\omega)$. They find $s$-wave superconductivity with an enhanced ratio $2 \Delta(0) / k_{B} T_{c}$ and a sharply decreasing $1 / T_{1} T$ below $T_{c}$ rather than a coherence peak.

In this paper we shall show, without referring to any specific theory about the origin of the superconductivity, that the superconducting state is very much affected by the normal-state MFL behavior. We demonstrate this by analyzing the effect of an additional attractive BCS-type potential on a system in which the dominant scattering causes MFL behavior. The transition temperature $T_{c}$ for this case can be determined from the ladder instability of the normal state. Below $T_{c}$, when the gap opens up, the suppression of the (electronic) bosonic mode is modeled by a scattering rate that decreases with decreasing temperature. The gap at zero temperature, $\Delta(0)$, remains practically unaffected by the MFL behavior above $T_{c}$ if the MFL scattering is sufficiently suppressed at $T=0$. Consequently, the ratio $2 \Delta(0) / k_{B} T_{c}$ is enhanced even for a weak-coupling superconductor, and the gap opens up rapidly; even a discontinuous first-order transition at $T_{c}$ is possible. Furthermore, we find that this behavior of the gap together with the smearing of the singularity in the BCS density of states due to the MFL scattering suppresses the coherence peaks.

Although the dominant scattering leads to MFL behavior a weaker interaction can be the origin of the su- 
perconductivity if retardation effects play a role, i.e., if the different interactions involve different time scales. It is therefore quite possible that the anomalous properties of the superconducting state of the cuprates are mainly due to the fact that the pairing occurs in a strongly correlated system (with MFL behavior), and that they are only weakly related to the origin of the pairing. Indeed, our results demonstrate a large degree of insensitivity of the unusual superconducting properties to the pairing mechanism. This becomes especially apparent by comparing our results to those of Littlewood and Varma, ${ }^{9}$ which are very similar, but obtained with the MFL boson taken as the origin of the superconductivity.

\section{II. $2 \Delta(0) / k_{B} T_{c}$ AND SUPPRESSION OF $T_{c}$}

With the simple model form for the attractive BCS interaction (up to an energy $\omega_{0}$ ) $T_{c}$ can be determined from the ladder instability of the normal state. Here we neglect differences between the pair-formation temperature and the actual transition temperature. We take the normal-state propagators with the MFL self-energy,

$$
\Sigma(\mathbf{k}, \omega)=\lambda\left[\omega \ln \left(x / \omega_{c}\right)-i(\pi x / 2)\right] .
$$

Here $x=\max (|\omega|, T)$ and $\omega_{c}$ is a large cutoff scale, which is estimated to be of the order of $0.5 \mathrm{eV}$ from Raman scattering; ${ }^{1}$ also the infrared measurements of Rotter et al. ${ }^{10}$ which show a quasiparticle scattering rate, which is linear in $\omega$ up to frequencies of $3000 \mathrm{~cm}^{-1}(0.37$ $\mathrm{eV}$ ) indicate a cutoff of this order. The value of $\lambda$ can be determined from resistivity measurements. Taking from Ref. 11 the result of resistivity measurements up to $600 \mathrm{~K}$ on $\mathrm{Bi}_{2} \mathrm{Sr}_{2.2} \mathrm{Ca}_{0.8} \mathrm{Cu}_{2} \mathrm{O}_{8}$ that

$$
d \rho(T) / d T=0.46 \mu \Omega \mathrm{cm} / \mathrm{K},
$$

we find $\lambda=0.23$, assuming a plasma frequency of $1 \mathrm{eV}$. Though the value of $\lambda$ is certainly not the same for all high- $T_{c}$ materials, it is of this order of magnitude.

The temperature Green function in the normal state then is

$$
G\left(\mathbf{k}, i \omega_{n}\right)=\left[i \omega_{n}-\epsilon(\mathbf{k})-\Sigma\left(i \omega_{n}\right)\right]^{-1},
$$

where from analytic continuation of the marginal Fermi liquid $\Sigma(\mathbf{k}, \omega)$ it follows that

$$
\begin{aligned}
\Sigma\left(i \omega_{n}\right) & =-i \lambda T \arctan \left(\frac{T}{\omega_{n}}\right) \\
& -\frac{1}{2} i \lambda \omega_{n} \ln \left(\frac{\omega_{n}^{2}+\omega_{c}^{2}}{\omega_{n}^{2}+T^{2}}\right) .
\end{aligned}
$$

The pairing-instability occurs at the temperature where the particle-particle scattering amplitude

$$
\Gamma[\mathbf{k}, \omega, \mathbf{k}, \omega ;-\mathbf{k},-\omega,-\mathbf{k},-\omega)
$$

diverges (Fig. 1), ${ }^{12}$

$$
1=N(0) V T \sum_{\left|\omega_{n}\right|<\omega_{0}} \int d \epsilon(\mathbf{k}) G\left(-\mathbf{k},-i \omega_{n}\right) G\left(\mathbf{k}, i \omega_{n}\right),
$$

where $N(0)$ is the density of states at the Fermi energy and $V$ is the strength of the BCS-type interaction with a cutoff energy $\omega_{0}$. The $\epsilon(\mathbf{k})$ integration is straightforward, leading to

$$
1=2 N(0) \sum_{n=0}^{\omega_{0} / 2 \pi T-(1 / 2)}\left\{(2 n+1)\left[1+\frac{\lambda}{2} \ln \left[\frac{(2 n+1)^{2} \pi^{2} T^{2}+\omega_{c}^{2}}{(2 n+1)^{2} \pi^{2} T^{2}+T^{2}}\right]+\frac{\lambda}{(2 n+1) \pi} \arctan \left[\frac{1}{(2 n+1) \pi}\right]\right\}^{-1} .\right.
$$

The remaining sum over the Matsubara frequencies can, under the condition that $\omega_{0}<\omega_{c}$ or that $\lambda$ is sufficiently small, be approximated by

$$
\begin{aligned}
& 2 N(0) V \sum_{n=0}^{\omega_{0} / 2 \pi T-(1 / 2)} \frac{1}{(2 n+1)\left[1-\lambda \ln \left((2 n+1) \pi T / \omega_{c}\right)\right]} \\
& \approx 2 N(0) V \sum_{n=0}^{\omega_{0} / 2 \pi T-(1 / 2)} \frac{1}{(2 n+1)\left[1+\lambda \ln \left(\omega_{c} / \pi T\right)\right]}\left[1+\frac{\lambda \ln (2 n+1)}{1+\lambda \ln \left(\omega_{c} / \pi T\right)}\right] .
\end{aligned}
$$

Performing the sum in the first term and writing the second sum as an integral gives the instability condition, which can be written as a quadratic equation for $\ln \left(\omega_{0} / \pi T_{c}\right) \equiv X, A X^{2}+B X+C=0$, with the coefficients

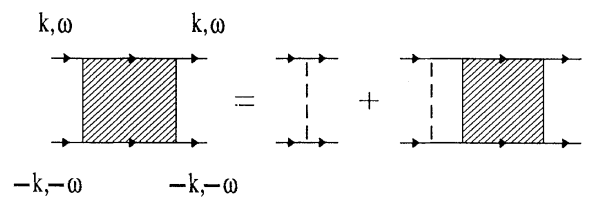

FIG. 1. The particle-particle ladder. The dashed lines denote simple BCS interactions, the propagators have a marginal Fermi liquid self-energy.

$$
\begin{aligned}
A \equiv & \frac{5 \lambda}{4}-\frac{\lambda^{2}}{N(0) V}, \\
B \equiv & -\frac{2 \lambda}{N(0) V}(1-\lambda \ln \alpha) \\
& -\lambda\left[\ln 2+\Psi\left[\frac{1}{2}\right]\right]+(1-\lambda \ln \alpha), \\
C \equiv & -\frac{1}{N(0) V}(1-\lambda \ln \alpha)^{2} \\
& -\left[\ln 2+\Psi\left[\frac{1}{2}\right]\right](1-\lambda \ln \alpha) .
\end{aligned}
$$


Here $\Psi(x)$ is the digamma function and $\alpha \equiv \omega_{0} / \omega_{c}<1$. The critical temperature then is found to be

$$
T_{c}(\lambda)=\frac{\omega_{0}}{\pi} \exp \left[\frac{B-\sqrt{B^{2}-4 A C}}{2 A}\right] .
$$

Taking for instance $\lambda=0.23, \omega_{0}=0.2 \mathrm{eV}, \omega_{c}=0.5 \mathrm{eV}$, and $N(0) V=0.5$ yields $T_{c}=91 \mathrm{~K}$. In the limit $\lambda \rightarrow 0$ one recovers the BCS critical temperature, $T_{c}^{\mathrm{BCS}}$ $=1.13 \omega_{0} \exp [-1 / N(0) V]$, which is higher than $T_{c}(\lambda>0)$. In Fig. 2 we have plotted $T_{c}(\lambda=0) / T_{c}\left(\lambda_{n s}\right)$ as a function of $\lambda_{n s}$ for different values of $N(0) V$ and with $\alpha=0.4$. One sees that the stronger the BCS coupling is, the smaller the relative suppression of $T_{c}$ due to the MFL scattering. Thus the critical temperature is suppressed as compared to the BCS value by the MFL behavior of the normal state. This is a natural consequence of the pair breaking caused by the inelastic MFL scattering.

An immediate consequence of the electronic origin of the MFL mode is that at low temperatures where the gap develops, the MFL mode itself will also develop a gap, and the low-energy scattering will be suppressed. ${ }^{9}$ The suppression of the quasiparticle scattering rate below $T_{c}$ can be described phenomenologically by $1 / \tau(T)$ $=[\lambda(T) \pi / 2] T$, where $\lambda$ is constant for $T>T_{c}$, indicated by $\lambda_{n s}$. As explained above we estimate $\lambda_{n s}=0.23$ from the experiment of Ref. 11. Upon entering the superconducting state $\lambda(T)$ decreases.

In case the MFL interaction is completely frozen out at zero temperature the zero temperature gap $\Delta(0)$ will not be affected by the MFL scattering and thus assumes the BCS value. Therefore, the ratio $2 \Delta(0) / k_{B} T_{c}$ increases entirely due to the effect of the MFL pair-breaking scattering on the transition temperature,

$$
\frac{2 \Delta(0)}{k_{B} T_{c}}=3.53 \frac{T_{c}(\lambda=0)}{T_{c}\left(\lambda_{n s}\right)} .
$$

In general, if the MFL mode is not entirely suppressed at zero temperature, a smaller ratio is obtained. When a possible small effect of a temperature-independent inelas-

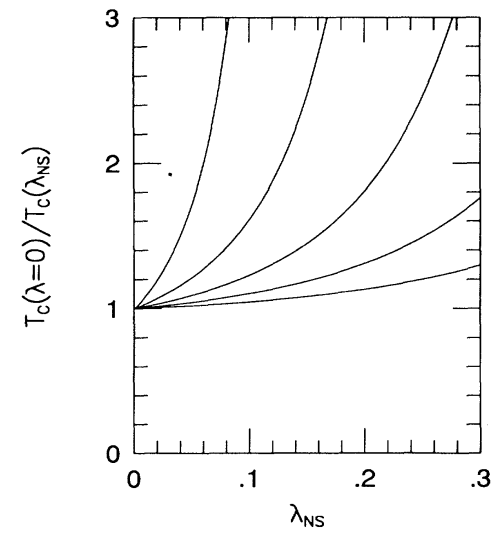

FIG. 2. $T_{c}(\lambda=0) / T_{c}\left(\lambda_{n s}\right)$ vs $\lambda_{n s}$. The different curves correspond, from top to bottom, with values of $N(0) V=0.3,0.45$, $0.6,0.75$, and 0.9 . tic scattering on the ratio $2 \Delta(0) / k_{B} T_{c}$ is neglected, one has

$$
\frac{2 \Delta(0)}{k_{B} T_{c}}=3.53 \frac{T_{c}(\lambda(T=0))}{T_{c}\left(\lambda_{n s}\right)} .
$$

Thus with separate origins of the anomalous normal-state properties (arising from the MFL polarizability) and the superconductivity (originating from the BCS model interaction) a situation arises that resembles the strongcoupling situation with an enhanced value of $2 \Delta(0) / k_{B} T_{c}$. The reason is that the inclusion of the MFL self-energy in the propagators of the particleparticle ladder describes the pair-breaking effects, which are suppressed at low temperatures. Such a suppression also occurs in the strong-coupling phonon situation, where the pair breaking due to thermal phonons has a relatively large effect on the transition temperature but only a small effect on the gap at zero temperature, where no thermal phonons are present.

In the extreme limit in which the quasiparticle scattering rate becomes negligible as soon as the gap opens up, the present model reduces to the BCS problem for all $T<T_{c}$. Then, $\Delta(T)$ follows a BCS dependence $\Delta_{\mathrm{BCS}}(\lambda=0, T)$ for all $T<T_{c}\left(\lambda_{n s}\right)$. The behavior of $\Delta_{\mathrm{BCS}}(\lambda=0, T)$ is indicated by the uppermost dashed curve in Fig. 3. Clearly, in this extreme limit, $\Delta$ jumps to a finite value at $T_{c}\left(\lambda_{n s}\right)$. In the general case in which the quasiparticle scattering rate remains nonvanishing below $T_{c}$, the opening up of the gap is less drastic, but still more
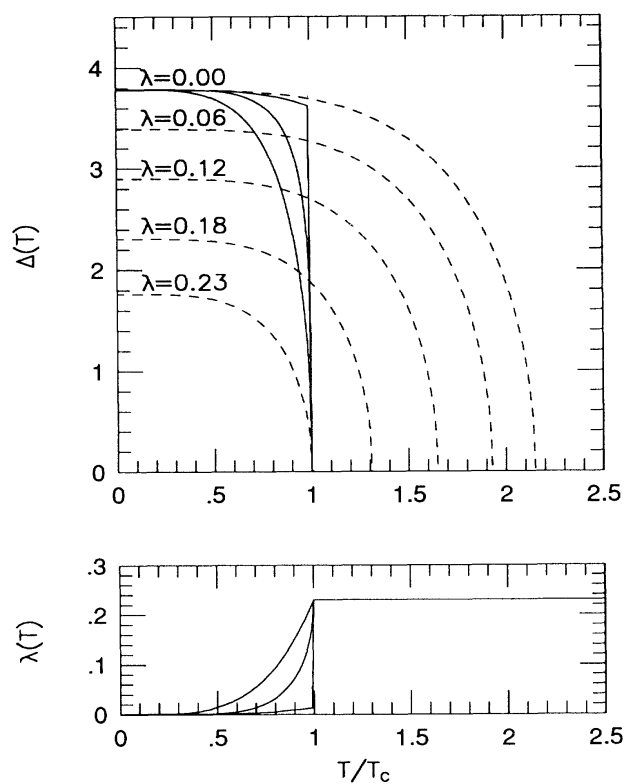

FIG. 3. The gap as a function of temperature (upper figure) with a temperature-dependent $\lambda$ (lower figure) with $2 \Delta(0) / k_{B} T_{c} \approx 7.8$. The curves in the lower figure correspond to curves in the upper figure: the lower the $\lambda(T)$ curve, the higher the $\Delta(T)$ curve. The dashed lines are BCS curves (thus with $\left.2 \Delta(0) / k_{B} T_{c}=3.53\right)$ through $T_{c}(\lambda)$, with the values of $\lambda$ given in the figure. We used $N(0) V=0.6, \omega_{c}=0.5 \mathrm{eV}$ and $\omega_{0}=0.2 \mathrm{eV}$. 
rapid than in the BCS case: the quicker $\lambda(T)$ decreases below $T_{c}$, the quicker the gap opens up, and vice versa. Although, in principle, the full complex gap equations have to be solved below $T_{c}$ to study this behavior, the general trend can be understood as follows. Consider a temperature-independent $\lambda$; for a given value of $\lambda$ the transition temperature $T_{c}(\lambda)$ is known from (8) to increase with decreasing $\lambda$. In a rough approximation, we can take for the temperature dependence of $\Delta(\lambda, T)$ for fixed $\lambda$ a BCS curve through $T_{c}(\lambda)$. Curves of this type are indicated by dashed lines in Fig. 3. (The ratios of the $T_{c}$ 's for $\lambda=0,0.06,0.12,0.18$, and 0.23 are taken from the $N(0) V=0.6$ curve in Fig. 2.) If we now consider a temperature-dependent $\lambda(T)$ as the upper two curves drawn in the lower part of Fig. 3, we obtain a gap that "scans" the constant- $\lambda$ curves between the $\lambda=0.23$ curve at $T_{c}$ and the $\lambda=0$ curve at $T=0$. Evidently this leads to a relatively rapid opening up of the gap and a larger ratio $2 \Delta(0) / k_{B} T_{c}$, which for the curve we have shown is about 7.8. In fact, if we take the scattering rate as a function of $\Delta(T)$ and $T$, rather than of $T$ only, the opening up of the gap and the decrease of the scattering rate even show a discontinuity at $T_{c}$, very much like the behavior discussed above in the limit $\lambda \rightarrow 0$ for all $T<T_{c}$. This discontinuity is found to be very large when we use

$$
\lambda\left(T<T_{c}\right) / \lambda_{n s}=2 /\{1+\exp [\Delta(T) / T]\} .
$$

(This ratio is the low frequency limit of the ultrasonic attenuation in BCS theory, i.e., a coherence-type-I suppression). Of course, it also leads to a discontinuity in $\lambda$ at $T_{c}$. Finite frequencies (and presumably also correlation effects leading to a vertex renormalization) might weaken this drastic behavior, leading to a smaller jump of the gap at $T_{c}$ and a remaining amount of inelastic scattering in the superconductive state. Experiments seem to agree that the temperature dependence of $\Delta(T)$ is very steep, if not discontinuous. ${ }^{4}$

\section{COHERENCE PEAKS}

BCS theory predicts coherence peaks in the nuclearspin-relaxation rate and the microwave conductivity as a function of temperature. These peaks appear at about $0.85 T_{c}$ and have a width of typically $0.3 T_{c}$. They reflect the properties of the quasiparticles and the singular nature of the density of states just above the gap, $N_{s}(\omega)=N_{n}(0) \omega / \sqrt{\omega^{2}-|\Delta|^{2}}$. In the high-temperature superconductors these coherence peaks are absent. ${ }^{2}$ In Eliashberg strong-coupling theory (with an $\alpha^{2} F(\omega)$ from the MFL mode ${ }^{8,9}$ or from phonons ${ }^{13-15}$ ) it is found that they may disappear for sufficiently strong coupling.

With $2 \Delta(0) / k_{B} T_{c}$ large, the coherence peaks become narrower and are located closer to $T_{c}$ than in the BCS case. This is due to the same effect that leads to the steep behavior of the gap: As the parameter $\lambda$ changes upon entering the superconductive state a scan of the BCS curves between the $\lambda=0.23$ curve at $T_{c}$ and the $\lambda=0$ curve at $T=0$ (as described above for the gap) is made, as shown in Fig. 4. This yields a narrow coherence peak.

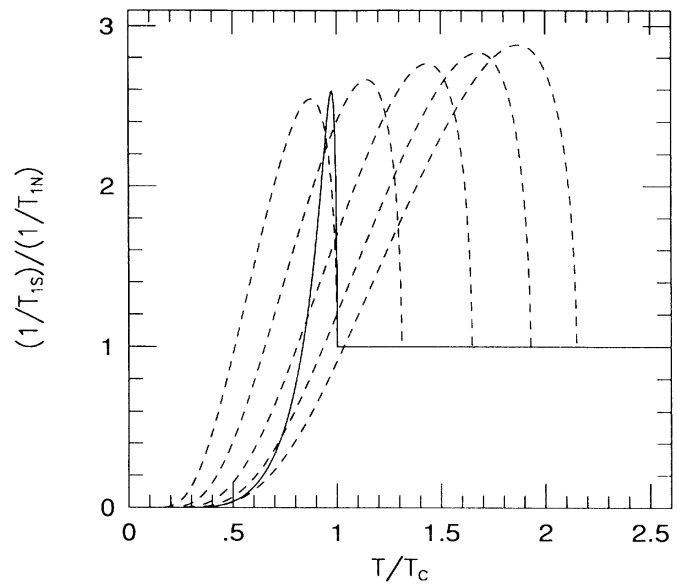

FIG. 4. Coherence peak (solid curve) with a temperaturedependent quasiparticle lifetime $1 / \tau=[\lambda(T) \pi / 2] T, \lambda(T)$ taken as the middle $\lambda(T)$ curve in Fig. 3, but without broadening of the density of states taken into account. The dashed curves are BCS coherence peaks, $T_{c}$ determined by (8) with, from right to left, $\lambda=0,0.06,0.12,0.18$, and 0.23 .

By using directly the steep gap, one also finds this narrow coherence peak. With $\lambda$ being a function of $\Delta(T)$, as was considered above, the coherence peak disappears completely when the discontinuity of the gap, and thus of $\lambda$ at $T_{c}$ is sufficiently large. If $\lambda$ changes continuously at $T_{c}$, always a narrow peak remains. The same mechanism leads to a more rapid suppression of the ultrasonic attenuation rate below $T_{c}$, as shown in Fig. 5.

Close to $T_{c}$, in the region of the narrow peak, the inelastic scattering is still active. This scattering, in addition to the BCS interaction, has the effect of smearing the square-root singularity in the BCS density of states. With a quasiparticle lifetime of the order of $0.2 \times 10^{-13} \mathrm{sec}$ close to $T_{c}$, as given by Bonn et al., ${ }^{6}$ the spectral

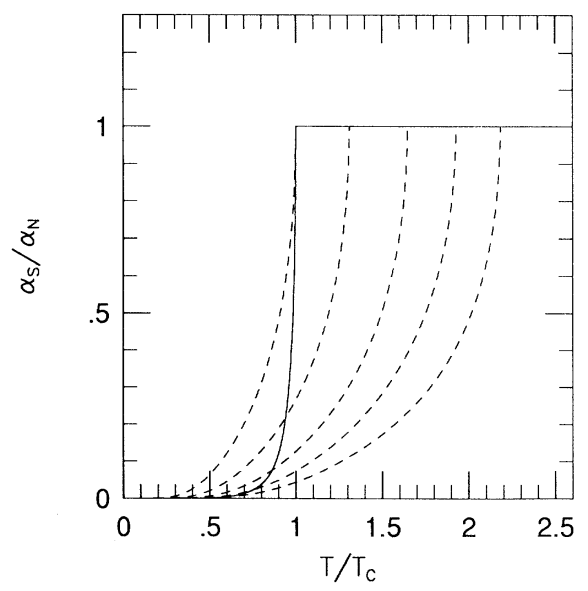

FIG. 5. The ultrasonic attenuation rate in the superconducting state normalized to the normal-state rate. The same values of $\lambda$ as in Fig. 4 are used. 
broadening near $T_{c}$ is of the order of the zerotemperature gap in the cuprates. At lower temperatures the broadening is strongly reduced due to the suppression of the inelastic scattering.

The smearing of the BCS density of states due to the MFL scattering is shown in Fig. 6. It is calculated from the spectral function of a BCS superconductor, with an imaginary part of the self-energy $1 / \tau=[\lambda(T) \pi / 2] T$, i.e.,

$$
\begin{aligned}
& \frac{N_{s}(\omega)}{N_{n}(0)}=\frac{1}{2 \pi i} \int d \epsilon(\mathbf{k})[ G(\mathbf{k}, z \rightarrow \omega-i / \tau) \\
&-G(\mathbf{k}, z \rightarrow \omega+i / \tau)],
\end{aligned}
$$

where

$$
G(\mathbf{k}, z)=\frac{z+\epsilon(\mathbf{k})}{z^{2}-E(\mathbf{k})^{2}}
$$

and $E(\mathbf{k})=\sqrt{\epsilon(\mathbf{k})^{2}+|\Delta|^{2}}$. The integral can be evaluated
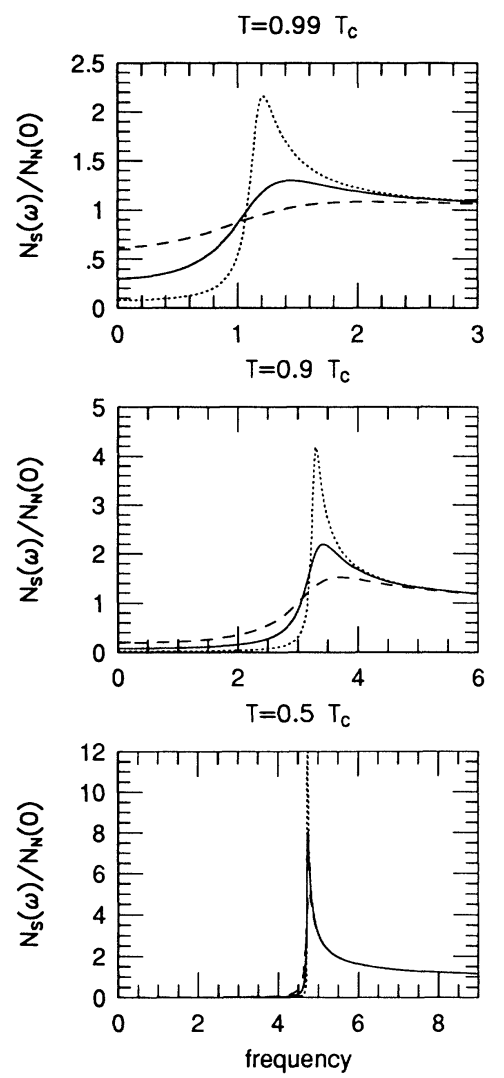

FIG. 6. The density of states in the superconducting state with the MFL interaction at different temperatures. At low temperatures the MFL interaction is suppressed and the BCS square-root singularity is recovered. At higher temperatures the MFL interaction is stronger, and the smearing of the singularity stronger. The dotted curve is obtained with a scattering rate of $0.02 \times \Delta(0)$ at $T_{c}$, the solid line with a scattering rate of $0.05 \times \Delta(0)$ at $T_{c}$ and the dashed line with a scattering rate of $0.2 \times \Delta(0)$ at $T_{c}$. We used a decrease of the scattering rate as indicated by the solid curve in Fig. 3, i.e., $\lambda(T)=\left(T / T_{c}\right)^{4} \lambda_{n s}$ below $T_{c}$. Even our largest scattering rate is modest compared to the one given by Bonn et al. (Ref. 6).

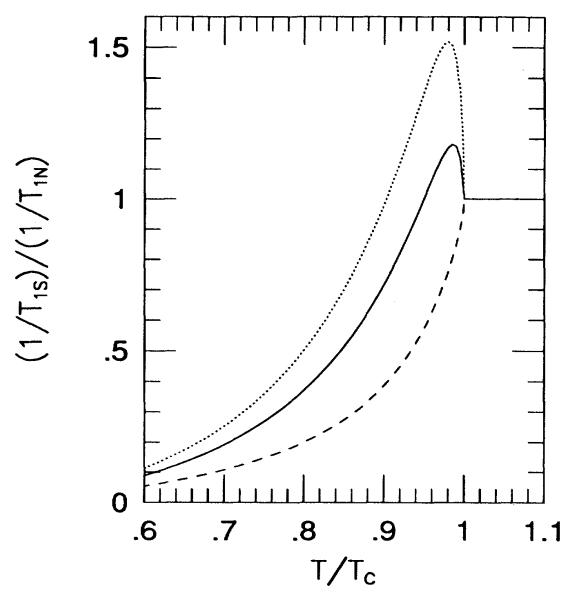

FIG. 7. The nuclear spin relaxation rate $1 / T_{1}$ normalized to the normal-state value with broadening of the density of states taken into account. The scattering rates are the same as in Fig. 6 .

exactly by contour integration:

$$
\begin{aligned}
\frac{N_{s}(\omega)}{N_{n}(0)}=\frac{1}{2 \tau \gamma \sqrt{\beta^{2}+\gamma^{2}}}[ & \left(\beta+\alpha^{2}\right)\left(2 \sqrt{\beta^{2}+\gamma^{2}}+2 \beta\right)^{1 / 2} \\
& \left.+\gamma\left(2 \sqrt{\beta^{2}+\gamma^{2}}-2 \beta\right)^{1 / 2}\right]
\end{aligned}
$$

where $\alpha^{2}=\omega^{2}+\Delta^{2}+(1 / \tau)^{2}, \quad \beta=\omega^{2}-\Delta^{2}-(1 / \tau)^{2}$, and $\gamma=2 \omega / \tau$. The quasiparticle spectrum obtained in this way is always gapless in case of nonvanishing MFL scattering, with the zero frequency value

$$
N_{s}(\omega \rightarrow 0, T) / N_{n}(0)=1 / \sqrt{1+\Delta(T)^{2} \tau(T)^{2}} .
$$

In Fig. 6 we have shown $N_{s}(\omega) / N_{n}(0)$ for different temperatures and different scattering rates at $T_{c}$. The temperature dependence of the MFL scattering rate shows up in the decreased amount of broadening at low temperatures.

Such a broadening of the square root singularity in the density of states, which occurs especially near $T_{c}$, where the narrow coherence peak is located, suppresses the peak largely or totally, as is shown in Fig. 7. This suppression even occurs for scattering rates that are smaller by a factor of two than quoted above.

\section{CONCLUSIONS}

We have considered a system of electrons with an (unidentified) dominant scattering mechanism that leads to marginal Fermi-liquid behavior and a BCS model interaction, that causes superconductivity. In absence of the MFL scattering the system would be a BCS superconductor. The MFL scattering is a pair-breaking process that lowers the critical temperature, which we calculated from the divergence of the particle-particle scattering amplitude. If the MFL mode is sufficiently suppressed below $T_{c}$ it has little effect on the zero-temperature gap. Consequently, $2 \Delta(0) / k_{B} T_{c}$ is enhanced. The gap opens up relatively rapidly, possibly even with a discontinuity at $T_{c}$. The inelastic MFL scattering also broadens the 
square-root singularity in the density of states which, together with the enhanced value of $2 \Delta(0) / k_{B} T_{c}$, leads to a suppression of the coherence peaks. Type-I-coherence behavior as followed by the ultrasonic attenuation is more rapidly suppressed below $T_{c}$ than in the BCS case. This also applies for the penetration depth. Our simple description embodies the relevant features of the Eliashberg strong-coupling approach: pair breaking and broadening of the density of states due to inelastic scattering, and therefore indeed recovers many of the results obtained by Littlewood and Varma. ${ }^{9}$ However, in our case the interaction that causes superconductivity is of BCS type, rather than the MFL mode. Our results also are similar to those obtained in numerical calculations by Bandte, Hertel, and Appel. ${ }^{16}$

\section{ACKNOWLEDGMENTS}

M.H. and F.V. gratefully acknowledge financial support by the Netherlands Foundation for Fundamental Research on Matter (FOM).
*Address after 1 September 1993: Department of Physics, Rutgers University, P.O. Box 849, Piscataway, New Jersey 08854.

${ }^{1}$ C. M. Varma, P. B. Littlewood, S. Schmitt-Rink, E. Abrahams, and A. E. Ruckenstein, Phys. Rev. Lett. 63, 1996 (1989).

${ }^{2}$ W. W. Warren, Jr., R E. Walstedt, G. F. Brennert, G. P. Espinosa, and J. P. Remeika, Phys. Rev. Lett. 59, 1860 (1987); P. C. Hammel, M. Takigawa, R. H. Heffner, Z. Fisk, and K. C. Ott, ibid. 63, 1992 (1989).

${ }^{3}$ G. Grüner, in Phenomenology and Applications of HighTemperature Superconductors, edited by K. S. Bedell et al. (Addison Wesley, Reading, MA, 1992).

${ }^{4}$ M. Nuss, P. M. Mankiewicz, M. L. O’Malley, E. H. Westerwick, and P. B. Littlewood, Phys. Rev. Lett. 63, 3305 (1991); M. L. Horbach and W. van Saarloos, Phys. Rev. B 46, 432 (1992).

${ }^{5}$ Z. Schlesinger, R. T. Collins, F. Holtzberg, C. Feild, G. Koren, and A. Gupta, Phys. Rev. B 41, 11237 (1990); M. Boekholt, M. Hoffmann, and G. Güntherodt, Physica C 175, 127 (1991); Yonghong Li, Jin Lin Huang, and C. M. Lieber, Phys. Rev. Lett. 68, 3240 (1992).

${ }^{6}$ D. A. Bonn, P. Dosanjh, R. Liang, and W. N. Hardy, Phys.
Rev. Lett. 68, 2390 (1992).

${ }^{7} \mathrm{P}$. W. Anderson, in Frontiers and Borderlines in Many-Particle Physics, edited by R. A. Broglia and J. R. Schrieffer (NorthHolland, Amsterdam, 1988).

${ }^{8}$ Y. Kuroda and C. M. Varma, Phys. Rev. B 42, 8619 (1990).

${ }^{9}$ P. B. Littlewood and C. M. Varma, Phys. Rev. B 46, 405 (1992); J. Appl. Phys. 69, 4979 (1991).

${ }^{10}$ L. D. Rotter, Z. Schlesinger, R. T. Collins, F. Holtzberg, C. Feild, U. W. Welp, G. W. Crabtree, J. Z. Liu, Y. Fang, K. G. Vandervoort, and S. Fleshler, Phys. Rev. Lett. 67, 2741 (1991).

${ }^{11}$ S. Martin, A. T. Fiory, R. M. Fleming, L. F. Schneemeyer, and J. V. Waszczak, Phys. Rev. Lett. 60, 2194 (1988).

${ }^{12}$ A. A. Abrikosov, L. P. Gorkov, and I. E. Dzyaloshinski, Methods of Quantum Field Theory in Statistical Physics (Dover, New York, 1975).

${ }^{13}$ R. Akis and J. P. Carbotte, Solid State Commun. 78, 393 (1991).

${ }^{14}$ P. B. Allen and D. Rainer, Nature (London) 349, 396 (1991).

${ }^{15}$ L. Coffey, Phys. Rev. Lett. 64, 1071 (1990).

${ }^{16}$ C. Bandte, P. Hertel, and J. Appel, Phys. Rev. B 45, 8026 (1992); C. Bandte, ibid. 47, 5473 (1993). 